\title{
A Simple Synthesis Route for Selectively Methylated $\beta$-Cyclodextrin Using a Copper Complex Sandwich Protecting Strategy
}

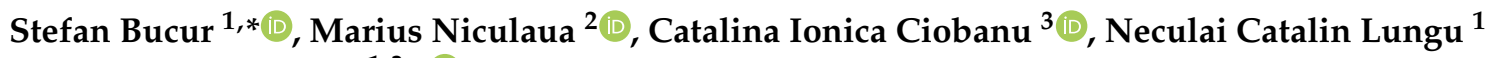 \\ and Ionel Mangalagiu $1,3, *$ (i)
}

1 Faculty of Chemistry, Alexandru Ioan Cuza University of Iasi, 700506 Iasi, Romania; lungu@uaic.ro

2 Research Center of Oenology, Romanian Academy_Iași Division, 700505 Iași, Romania; niculaua@acadiasi.ro

3 Institute of Interdisciplinary Research-CERNESIM Centre, Alexandru Ioan Cuza University of Iasi, 700506 Iași, Romania; catalina.ciobanu@uaic.ro

* Correspondence: bucurm.stefan@gmail.com or stefan.bucur@chem.uaic.ro (S.B.); ionelm@uaic.ro (I.M.)

check for

updates

Citation: Bucur, S.; Niculaua, M.; Ciobanu, C.I.; Lungu, N.C.;

Mangalagiu, I. A Simple Synthesis

Route for Selectively Methylated

$\beta$-Cyclodextrin Using a Copper

Complex Sandwich Protecting

Strategy. Molecules 2021, 26, 5669.

https://doi.org/10.3390/

molecules26185669

Academic Editor: Katia Martina

Received: 20 August 2021

Accepted: 13 September 2021

Published: 18 September 2021

Publisher's Note: MDPI stays neutral with regard to jurisdictional claims in published maps and institutional affiliations.

Copyright: (C) 2021 by the authors Licensee MDPI, Basel, Switzerland. This article is an open access article distributed under the terms and conditions of the Creative Commons Attribution (CC BY) license (https:/ / creativecommons.org/licenses/by/ $4.0 /)$.

\begin{abstract}
This communication reports a novel synthesis route for the preparation of monofunctionalized $\beta$-cyclodextrin in a single stage. The approach involves only the in-situ protection of secondary hydroxyl groups as an excellent alternative to the classical procedure involving a series of five steps of protection and deprotection of hydroxyl groups (both primary and secondary ones) belonging to $\beta$-cyclodextrin.
\end{abstract}

Keywords: cyclodextrin; selective primary side substitution; copper complex; control of the reactivity; one-pot reaction

\section{Introduction and Current Status of the Subject}

Cyclodextrin (CD) research has increased exponentially during the last fifty years. Currently, the number of derivatives for all three natural CDs is in the range of several thousands. Due to this wide variety of derivatives, one can wonder how these functional groups affect the general properties of CDs and what specific site should be changed for a particular application; for example, whether an increased water solubility or greater stability of the guest in the CD cavity is desired. The answer was partially given by Wenz [1], when through isothermal titration microcalorimetry, he established the binding constants of 4-tert-butylbenzoate and adamantane-1-carboxylate with all methylated $\beta$ cyclodextrin derivatives. These results reveal that by blocking the primary hydroxyl side of $\beta$-cyclodextrin ( $\beta$-CD), there is not only the benefit of increased water solubility, that all methylated derivatives display, but the maximum binding constant for the inclusion complexes in this series of methylated $\beta$-CDs can also be obtained. Although a complete change of all 21 hydroxy groups is relatively easy to perform in one step and the yield is quantitative, as demonstrated for per-O-methylated cyclodextrins using Haworth [2] and Irvine-Purdie methylation [3,4] or per-O-(2-hydroxypropyl) cyclodextrins [5], selective primary side derivatization is still in the early development stages, with one notable exception being the modified Appel reaction [6]. This reaction is used as the first step to synthesize per-6-halogeno-per-6-deoxy cyclodextrins, the $\gamma$-cyclodextrin variant being the precursor for octakis[6-(2-carboxyethylthio)-6-deoxy]- $\gamma$-cyclodextrin sodium salt also known as Sugammadex [7], one of the most successful cyclodextrin derivatives. The success of Sugammadex to selectively remove only the general anesthetic, and thus being the first selective relaxant binding agent, it gives some hints about the increased practicality of primary-side-substituted cyclodextrins as a more potent guest carrier. Another path for the primary side substitution is carried out using the classical organic chemistry protocols of protecting groups and numerous steps until the final desired product is obtained. These protocols employ the use of tert-butyldimethylsilyl chloride for primary side protection 
followed by a wide variety of secondary side protection (methylation, acetylation, benzylation, etc.) [8] with subsequently improved variants [9] and a relatively recent work by the cyclodextrin specialized laboratory CycloLab [10]. Moreover, even though highly efficient steps are now available, the multiple-step reactions needed to achieve the result bring down the total yield of the reaction to a reported value ranging between $40 \%$ and $70 \%[9,10]$. All the above-mentioned syntheses, summarized in Figure 1 offer good but expensive commercial products.

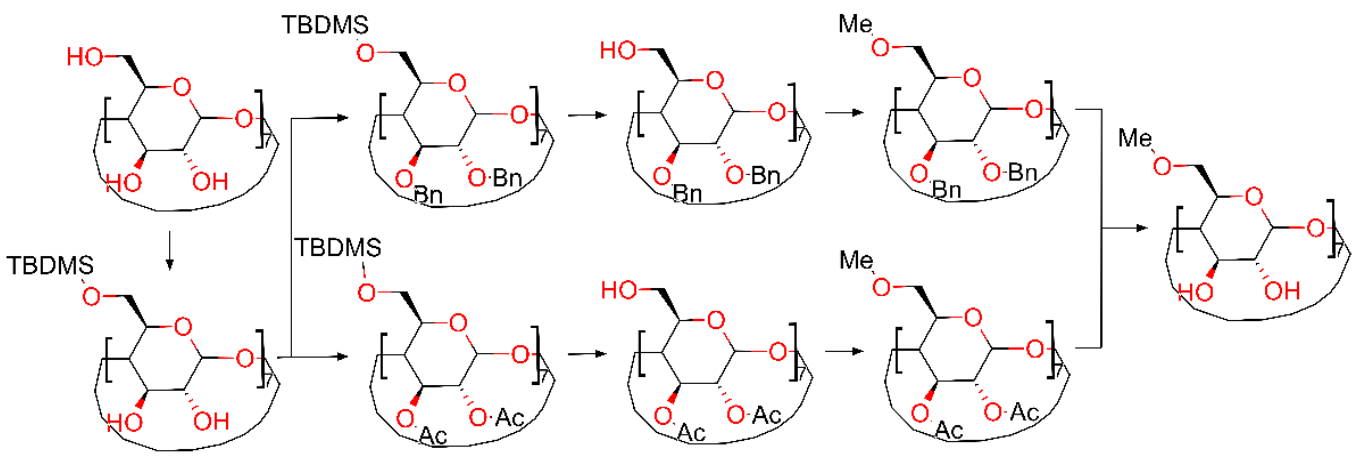

Figure 1. Classic organic chemistry protocol compiled from ref. [8-10].

One workaround employed in the industry is the use of cheap randomly substituted cyclodextrins. Their synthesis is almost always comprised of one step, followed by a simple purification workup. The drawback for these types of syntheses is the resulted mix of isomers characterized by an average degree of substitution (DS) following the normal Gaussian distribution. If the manufacturer does not follow good manufacturing practices, this normally distributed abundance of isomers can be affected, and for the same DS, one can have differently shaped distributions [11], affecting the reproducibility of proprieties of the end product. One of the easiest isomers to produce, and at the same time most studied, is the randomly substituted $\beta$-CD. For example, the phase-transfer catalysis method of methylation with dimethyl sulfate which produces a DS of $12.4 \div 13.2$ is called RAMEB [12]. A similar DS was obtained using $\mathrm{CH}_{3} \mathrm{I}$ [13], $\mathrm{CH}_{3} \mathrm{Cl}$ [14] or dimethyl carbonate, although the last reaction was performed in dimethylformamide solvent [15]. The influence of different reaction times and several strong bases on the DS and substitution pattern was investigated [16-18]. One of the earliest uses of copper chelates as temporary protection for selective acylation of aminoglycoside antibiotics was reported by Hanessian in 1978 [19]. Although pure cyclodextrins metal complexes are said to have only a few applications in Bellia's extensive review [20], in the same year, Masurier et al. used a copper(II)- $\beta$-cyclodextrin complex to synthesize 3-O-substituted $\beta$-cyclodextrin derivatives [21]. A sandwich copper- $\beta$-cyclodextrin was used to direct the tosylation only to primary hydroxyls groups, avoiding secondary side products [22]. With these sandwichtype complexes, the CDs are forming dimeric structures in which the secondary hydroxyl groups are coupled together by a ring of metal ions $[23,24]$. This complex is formed in one step and requires a basic medium to be stable, which is needed for the methylation reaction. The coordination of copper(II) ions, as proven by X-ray diffraction crystallography, is square-planar, with both secondary hydroxy groups of each glucose unit involved in the coordination. Herein, this study aims to provide a novel chemical shortcut of the classical procedure involving a series of five steps for the protection and deprotection of hydroxyl groups (both primary and secondary) belonging to $\beta$-cyclodextrin in a single stage, consisting of only in situ protection of secondary hydroxyl groups. This new synthesis route is exploiting the well-known ability of cyclodextrins to form coordination compounds with metals. In this respect, the copper(II) ion was chosen due to its $\mathrm{dsp}^{2}$ hybridization (by combining a $3 \mathrm{~d}_{x-y}{ }^{2}$ orbital with one $4 \mathrm{~s}$ and two $4 p$ orbitals, respectively) and its proper ionic radius which facilitates coordination in a square planar geometry with two secondary hydroxyl groups of a glucopyranose unit of a cyclodextrin on one side of the plane and 
two other similar groups of another cyclodextrin molecule oriented on the other side of the plane. The result is a sandwich structure with copper(II) ions in the middle, coordinating and blocking the reactivity of these functional groups. The coordination complex stability is enhanced when $\mathrm{pH}$ becomes increasingly alkaline, favoring a Haworth-type methylation by using dimethyl sulfate as an alkylating agent. The chemical reactions associated with this protection procedure are schematically illustrated in Figure 2.

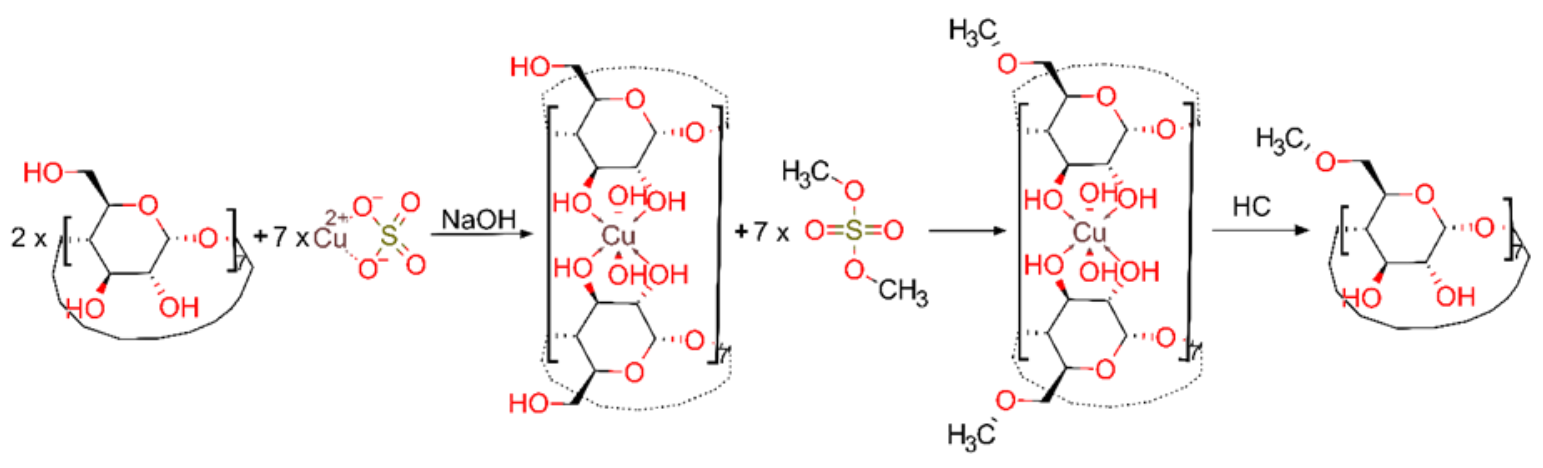

Figure 2. Proposed protection mechanism.

The main advantages of this new approach consist of shortening the number of purification steps-from five in the classical case to one in the proposed reaction route. Additionally, the synthesis takes place in water at room temperature by using only two reagents, with a practical and effective possibility regarding cupric ion recovery. In comparison with the classical route where a variety of solvents, reagents, catalysts and protective atmosphere is required with a non-negligible increase in the final price of the selectively methylated excipient at position $\mathrm{O}(6)$, the method proposed in this study has a huge advantage because of its simplicity and effectiveness. Furthermore, when comparing the proposed chemical synthesis with that of randomly methylated $\beta$-cyclodextrin (RAMEB), a single compulsory reagent is needed. This first step of complexation before methylation can guide the alkylation on the primary hydroxyl groups. Indeed, the reaction must be conducted with care to avoid overmethylation of the final compound if the reaction conditions are too energetic.

As far as the deprotection step is concerned, it is the same as the one used in the classical RAMEB synthesis. In addition, the neutralization of alkaline solution leads to a precipitate of cupric oxide which may be easily separated and removed from the reaction mixture via filtration.

In addition to the syntheses intended to optimize the ratio of $\beta-\mathrm{CD}: \mathrm{Me}_{2} \mathrm{SO}_{4}$ to obtain heptakis(6-O-methyl)- $\beta$-cyclodextrin $(\mathrm{M} \beta \mathrm{CD})$ and to prove the ability to protect secondary hydroxyl groups by forming the coordination complex $\beta-\mathrm{CD}_{2} \mathrm{Cu}_{7}(\mathrm{OH})_{14}$, several control syntheses (without cupric sulfate) were carried out to obtain RAMEB with different degrees of methylation. Comparative analyses of NMR spectra recorded for the fully methylated compound heptakis(2,3,6-tri-O-methyl)- $\beta$-cyclodextrin (TRIMEB) were performed to monitor the synthesized products.

\section{Results and Discussion}

M $\beta C D$ synthesis, presented in Figure 3, was performed with a $76 \%$ yield by using a novel chemical shortcut of the classical procedure involving a series of five steps for the protection and deprotection of hydroxyl groups (both primary and secondary) belonging to $\beta$-cyclodextrin in a single stage consisting of only in-situ protection of secondary hydroxyl groups. The results show that the new fabrication route is feasible and can evolve, after optimization to a revolutionary economical solution for replacing the classic five-step method. 


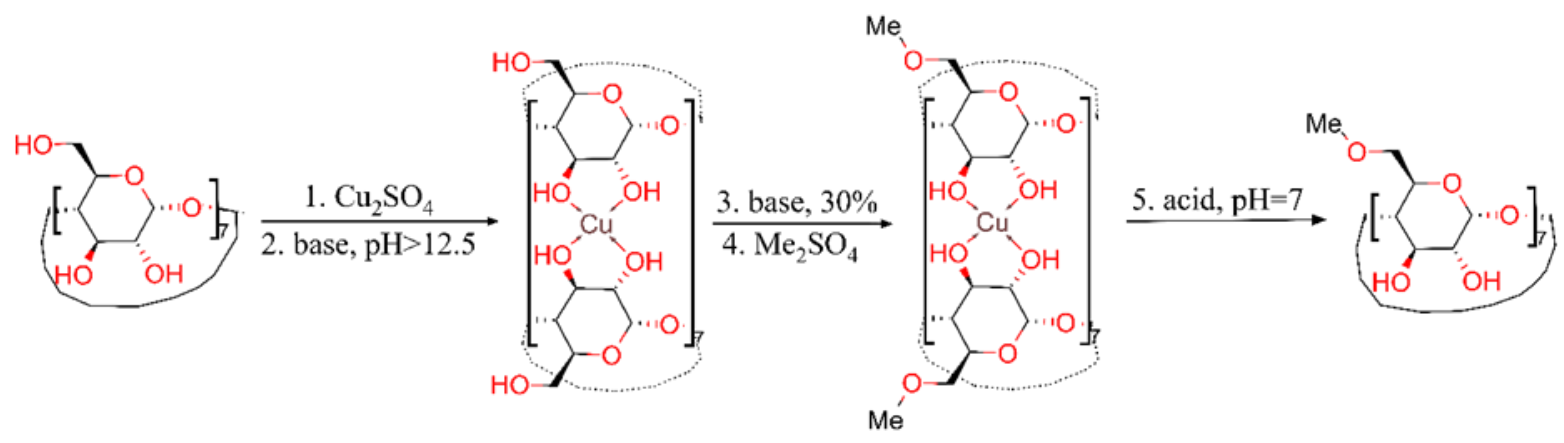

Figure 3. Synthesis of heptakis(6-O-methyl)- $\beta$-cyclodextrin $(\mathrm{M} \beta \mathrm{CD})$.

To prove the above, comparative analyses of NMR spectra recorded for the fully methylated compound heptakis(2,3,6-tri-O-methyl)- $\beta$-cyclodextrin (TRIMEB) were performed to monitor the synthesized products.

The ${ }^{1} \mathrm{H}-\mathrm{NMR}$ and ${ }^{13} \mathrm{C}-\mathrm{NMR}$ chemical shifts for $\beta$-CD and methylated $\beta$-CD in DMSO$\mathrm{d} 6$ as well as for the used references are presented in Tables 1 and 2.

Table 1. ${ }^{1} \mathrm{H}-\mathrm{NMR}$ chemical shifts for $\beta$-CD and methylated $\beta$-CD in DMSO-d6.

\begin{tabular}{cccccccc}
\hline & 2-OH & 3-OH & H1 & 6-OH & 3-O-Me & 2-O-Me & 6-O-Me \\
\hline$\beta-C D$ & 5.73 & 5.69 & 4.80 & 4.51 & - & - & - \\
M $\beta C D$ & 5.82 & 5.76 & 4.77 & 4.51 & - & - & 3.24 \\
M $\beta$ CD [9] & 5.80 & 5.72 & 4.77 & - & $3.59 *$ & $3.47 *$ & 3.24 \\
Reference sample & 5.83 & 5.74 & 4.77 & 4.46 & 3.69 & 3.40 & 3.23 \\
TRIMEB & - & - & 5.04 & - & 3.50 & 3.38 & 3.23 \\
TRIMEB [25] & - & - & 5.08 & - & 3.64 & 3.50 & 3.32 \\
DIMEB [25] & - & $\mathrm{N} / \mathrm{S}$ & 4.95 & - & - & 3.6 & 3.4 \\
\hline
\end{tabular}

* signals from the same reference but for heptakis(2,3-di-O-methyl)- $\beta$-cyclodextrin.

Table 2. ${ }^{13} \mathrm{C}-\mathrm{NMR}$ chemical shifts for $\beta$-CD and methylated $\beta$-CD in DMSO-d6.

\begin{tabular}{cccccccccc}
\hline & C1 & C4 & C3 & C2 & C5 & C6 & Me3 & Me2 & Me6 \\
\hline$\beta-C D$ & 102.14 & 81.73 & 73.27 & 72.58 & 72.24 & 60.15 & - & - & - \\
M $\beta C D$ & 102.24 & 82.26 & 73.06 & 72.44 & 70.31 & 70.94 & - & - & 52.87 \\
M CD [9] & 97.5 & 77.6 & 68.3 & 67.6 & 65.6 & 66.2 & $61.3 *$ & $58.6^{*}$ & 53.3 \\
Reference sample & 102.30 & 82.27 & 73.10 & 72.37 & 70.40 & 70.99 & 63.44 & 59.30 & 58.15 \\
TRIMEB [25] & 98.4 & 79.7 & 82.4 & 81.6 & 70.5 & 71.0 & 60.9 & 58.0 & 58.4 \\
DIMEB [25] & 101.3 & 82.1 & 73.1 & 83.6 & 70.9 & 71.4 & - & 60.3 & 58.7 \\
\hline
\end{tabular}

* signals from the same reference but for heptakis(2,3-di-O-methyl)- $\beta$-cyclodextrin.

The reference sample shows more or less the same substitution degree: in both ${ }^{1} \mathrm{H}$ and ${ }^{13} \mathrm{C}-\mathrm{NMR}$ spectra, the signals for all three methylated positions, 2, 3 and $6 \mathrm{O}-\mathrm{Me}$, are present. In the case of ${ }^{1} \mathrm{H}-\mathrm{NMR}$ for $\beta$-CD and methylated $\beta$-CD in DMSO-d6, the signals for 3-O-Me and 2-O-Me are missing. The reference sample, due to the mixture of methylated cyclodextrin, has multiple signals present in ${ }^{13} \mathrm{C}-\mathrm{NMR}$ spectra. The NMR recorded spectra for products and reference samples are presented in Supporting Information Figures S3-S8, S10, S11 and S13. MS analysis identified the $[\mathrm{M}+\mathrm{Na}]^{+}$adduct ions as normal isomer distributions for the sample methylated in the presence of $\mathrm{Cu}^{2+}$ ions. Complete MS results are presented in the Supporting Information Figures S9 and S12.

\section{Materials and Methods}

Reagents: $\beta$-cyclodextrin ( $\geq 95.0 \%$, Wacker Chemie AG, Munich, Germany) was vacuum-dried before use, copper sulfate (for analysis, $>99 \%$, Chemical Company SA, Iasi, Romania), sodium hydroxide (reagent grade, $\geq 98 \%$, pellets, Sigma-Aldrich/Merck KGaA, Darmstadt, Germany), dimethyl sulfate (puriss. p.a., $\geq 99.8 \%$, Sigma-Aldrich/Merck KGaA, Darmstadt, Germany), N,N-dimethylformamide (Reagent Plus, $\geq 99 \%$, SigmaAldrich/Merck KGaA, Darmstadt, Germany), ammonia (for analysis, min. 25\%, Chemical 
Company SA, Iasi, Romania), acetone (for analysis, $>99 \%$, Chemical Company SA, Iasi, Romania), ethanol (for analysis, $>96 \%$, Chemical Company SA, Iasi, Romania), chloroform (for analysis, $>98.5 \%$, Chemical Company SA, Iasi, Romania).

The syntheses were performed as follows:

Heptakis(6-O-methyl)- $\beta$-cyclodextrin synthesis $(\mathrm{M} \beta \mathrm{CD})$ was performed using $1 \mathrm{mmol}$ (1.135 g) $\beta$-CD and $4 \mathrm{mmol} \mathrm{CuSO}_{4}(0.9987 \mathrm{~g})$ dissolved in $100 \mathrm{~mL} \mathrm{H}_{2} \mathrm{O}$. To this solution, we added $50 \mathrm{~mL}$ of cold concentrated $\mathrm{NaOH}$ solution to reach $30 \%$ in the final volume. To this dark blue solution, $8 \mathrm{~mL}$ of $\mathrm{Me}_{2} \mathrm{SO}_{4}$ was slowly added dropwise from a dropping funnel. The reaction was continuously stirred for another $24 \mathrm{~h}$. At the end of the reaction time, the mixture was neutralized, and the precipitate was filtered on a G4 sintered glass funnel and washed with $3 \times 15 \mathrm{~mL} \mathrm{H}_{2} \mathrm{O}$. The solution was then concentrated to the minimum amount of water and the final product was solvent-extracted with $3 \times 150 \mathrm{~mL}$ $\mathrm{CHCl}_{3}$. The yield of $76 \%$ is strongly linked to the efficiency of this extraction so it might be improved, given the quantitative theoretical yield of this methylation reaction. RAMEB (reference sample) was synthesized using the same quantities and procedures as described above, but without adding copper sulfate to the reaction mixture, resulting in an $85 \%$ yield. Heptakis(2,3,6-tri-O-methyl)- $\beta$-cyclodextrin synthesis (TRIMEB) was performed using $5 \mathrm{mmol}(5.675 \mathrm{~g}) \beta$-CD dissolved in $150 \mathrm{~mL}$ of dry DMF. To this solution, we added $21 \mathrm{~g}$ of $\mathrm{NaOH}$ (1.25 equivalent) and after partial dissolution, $40 \mathrm{~mL}$ of $\mathrm{Me}_{2} \mathrm{SO}_{4}$ (1.25 equivalent) was slowly added dropwise from a dropping funnel. The reaction was continuously stirred for $48 \mathrm{~h}$. At the end of the reaction time, we decomposed the excess dimethyl sulfate with $50 \mathrm{~mL} \mathrm{NH}_{4} \mathrm{OH}$, and after $4 \mathrm{~h}$, the solvent and water were removed in the vacuum. A continuous solid-liquid extraction with chloroform was used to obtain the product with a $90 \%$ yield.

The NMR experiments were carried out on a Bruker Avance III $500 \mathrm{MHz}$ spectrometer operating at $500 \mathrm{MHz}$ for ${ }^{1} \mathrm{H}$ and $125 \mathrm{MHz}$ for ${ }^{13} \mathrm{C}$. Chemical shifts $(\delta)$ were reported in parts per million (ppm) using the solvent peak as the internal reference (DMSO-d6: $2.50 \mathrm{ppm}$ ). NMR data were processed and analyzed using BrukerTopSpin3.2. software. All NMR experiments were performed according to the scientific literature and can be found in the Supplementary Material.

Matrix-assisted laser desorption/ionization time of flight (MALDI-TOF) mass spectrometry experiments were carried out on Shimadzu AXIMA Performance and operated in high-resolution reflectron mode using $\alpha$-Cyano-4-hydroxycinnamic acid as the matrix.

\section{Conclusions}

This study provides a novel chemical shortcut of the classical procedure involving a series of five steps of protection and deprotection for hydroxyl groups (both primary and secondary) belonging to $\beta$-cyclodextrin in a single stage consisting of only the in situ protection of secondary hydroxyl groups. A yield of $76 \%$ for $M \beta C D$, strongly linked to the efficiency of the extraction, was obtained. It might be improved, given the quantitative theoretical yield of this methylation reaction. RAMEB (reference sample) synthesized using the same reaction conditions resulted in an $85 \%$ yield. TRIMEB synthesis was performed with a $90 \%$ yield. These results show that the proposed fabrication route is feasible and can evolve, after optimization to a revolutionary economical solution for replacing the five-step classic method.

Supplementary Materials: The following are available online, Figures S1-S13: NMR and MALDITOF Spectra.

Author Contributions: S.B., conceptualization, methodology, measurements, data analysis, validation, writing manuscript and revisions; M.N. MS and MALDI-TOF experiments, data analysis, validation; C.I.C., NMR experiments, data analysis, validation; N.C.L., data analysis, validation, supervision, resources; I.M., data analysis, validation, supervision, resources. All authors have read and agreed to the published version of the manuscript. 
Funding: This work was supported by a grant of the Romanian Ministry of Education and Research, CNCS-UEFISCDI, project number PN-III-P4-ID-PCE-2020-0371, within PNCDI III.

Institutional Review Board Statement: Not applicable.

Informed Consent Statement: Not applicable.

Data Availability Statement: The raw and processed data required to reproduce these findings cannot be shared at this time due to technical or time limitations. The raw and processed data will be provided upon reasonable request to anyone interested anytime, by the corresponding author.

Acknowledgments: Acknowledgment to the infrastructure support from Operational Program Competitiveness 2014-2020, Axis 1, under POC/448/1/1 Research infrastructure projects for public R\&D institutions/Sections F 2018, through the Research Center with Integrated Techniques for Atmospheric Aerosol Investigation in Romania (RECENT AIR) project, under grant agreement MySMIS no. 127324. The authors are thankful to UEFISCDI Bucharest, Romania, project PN-III-P11.1-TE-2016-1205, for financial support, and to CERNESIM Centre, for infrastructure used. Special thanks to Marin Micut from the University of Bucharest, Chemistry Faculty and Mirela Suchea from IMT Bucharest for the constructive discussions and advice.

Conflicts of Interest: The authors declare no conflict of interest.

\section{References}

1. Wenz, G. Influence of intramolecular hydrogen bonds on the binding potential of methylated $\beta$-cyclodextrin derivatives. Beilstein J. Org. Chem. 2012, 8, 1890-1895. [CrossRef]

2. Szejtli, J.; Lipták, A.; Jodál, I.; Fügedi, P.; Nánási, P.; Neszmélyi, A. Synthesis and ${ }^{13}$ C-NMR spectroscopy of methylated beta-cyclodextrins. Starch Stärke 1980, 32, 165-169. [CrossRef]

3. Nakazono, K.; Takashima, T.; Arai, T.; Koyama, Y.; Takata, T. High-yield one-pot synthesis of permethylated alpha-cyclodextrinbased polyrotaxane in hydrocarbon solvent through an efficient heterogeneous reaction. Macromolecules 2010, 43, 691-696. [CrossRef]

4. Stefanache, A.; Silion, M.; Stoica, I.; Fifere, A.; Harabagiu, V.; Farcas, A. Poly[2,7-(9,9-dioctylfluorene)-alt-(5,5'-bithiophene/permethylated beta-cyclodextrin)] main-chain polyrotaxane: Synthesis, characterization and surface morphology. Eur. Polym. J. 2014, 50, 223-234. [CrossRef]

5. Trotta, F.; Costa, L.; Giancarlo, C. A new easy access to enantiomerically pure 2,2'-dihydroxy-1,1'-binaphthyl. J. Incl. Phenom. Macrocycl. Chem. 2002, 44, 341-344. [CrossRef]

6. Ashton, P.R.; Königer, R.; Stoddart, J.F.; Alker, D.; Harding, V.D. Amino acid derivatives of $\beta$-cyclodextrin. J. Org. Chem. 1996, 61, 903-908. [CrossRef]

7. Bom, A.; Bradley, M.; Cameron, K.; Clark, J.K.; van Egmond, J.; Feilden, H.; MacLean, E.J.; Muir, A.W.; Palin, R.; Rees, D.C.; et al. A novel concept of reversing neuromuscular block: Chemical encapsulation of rocuronium bromide by a cyclodextrin-based synthetic host. Angew. Chem. Int. Ed. 2002, 41, 265-270. [CrossRef]

8. Takeo, K.; Mitoh, H.; Uemura, K. Selective chemical modification of cyclomalto- oligosaccharides via tert-butyldimethylsilylation. Carbohydr. Res. 1989, 187, 203-221. [CrossRef]

9. Uccello-Barretta, G.; Sicoli, G.; Balzano, F.; Salvadori, P. NMR spectroscopy: A powerful tool for detecting the conformational features of symmetrical persubstituted mixed cyclomaltoheptaoses ( $\beta$-cyclodextrins). Carbohydr. Res. 2005, 340, $271-281$. [CrossRef]

10. Bálint, M.; Darcsi, A.; Benkovics, G.; Varga, E.; Malanga, M.; Béni, S. Synthesis of the chiral selector heptakis(6-O-methyl)- $\beta$ cyclodextrin by phase-transfer catalysis and hydrazine-mediated transfer-hydrogenation. Electrophoresis 2019, 40, 1941-1950. [CrossRef]

11. Estrada, R.I.I.I.; Vigh, G. Comparison of charge state distribution in commercially available sulfated cyclodextrins used as chiral resolving agents in capillary electrophoresis. J. Chromatogr. A 2012, 1226, 24-30. [CrossRef] [PubMed]

12. Bakó, P.; Fenichel, L.; Töke, L.; Szente, L.; Szejtli, J. Methylation of cyclodextrins by phase-transfer catalysis. J. Incl. Phenom. Mol. Recognit. Chem. 1994, 18, 307-314. [CrossRef]

13. Wimmer, T. Process for the Preparation of Alkylated Cyclodextrin Derivatives, the Methylated Cyclodextrin Derivatives Obtainable from This Process and the Use of the Products. Patent DE4333598 (A1), 5 April 1995.

14. Cui, Y.; Wang, C.; Mao, J.; Yu, Y. A facile and practical approach to randomly methylated betacyclodextrin. J. Chem. Technol. Biotechnol. 2010, 85, 248-251. [CrossRef]

15. Gan, Y.; Zhang, Y.; Xiao, C.; Zhou, C.; Zhao, Y. A novel preparation of methyl-beta-cyclodextrin from dimethyl carbonate and beta-cyclodextrin. Carbohydr. Res. 2011, 346, 389-392. [CrossRef]

16. Pitha, J.; Milecki, J.; Fales, H.; Pannell, L.; Uekama, K. Hydroxypropyl-beta-cyclodextrin-preparation and characterization-effects on solubility of drugs. Int. J. Pharm. 1986, 29, 73-82. [CrossRef] 
17. Rao, C.T.; Pitha, J.; Lindberg, B.; Lindberg, J. Distribution of substituents in O-(2-hydroxypropyl) derivatives of cyclomaltooligosaccharides (cyclodextrins): Influence of increasing substitution, of the base used in the preparation, and of macrocyclic size. Carbohydr. Res. 1992, 223, 99-107. [CrossRef]

18. Yuan, C.; Liu, B.; Liu, H. Characterization of hydroxypropyl-beta-cyclodextrins with different substitution patterns via FTIR, GC-MS, and TG-DTA. Carbohydr. Polym. 2015, 118, 36-40. [CrossRef] [PubMed]

19. Hanessian, S.; Patil, G. Aminoglycoside antibiotics-A method for selective N-acylation based on the temporary protection of amino alcohol functions as copper chelates. Tetrahedron. Lett. 1978, 12, 1035-1038. [CrossRef]

20. Bellia, F.; La Mendola, D.; Pedone, C.; Rizzarelli, E.; Saviano, M.; Vecchio, G. Selectively functionalized cyclodextrins and their metal complexes. Chem. Soc. Rev. 2009, 38, 2756-2781. [CrossRef]

21. Masurier, N.; Lafont, O.; Le Provost, R.; Lesur, D.; Masson, P.; Djedaïni-Pilard, F.; Estour, F. Regioselective access to 3I-Osubstituted- $\beta$-cyclodextrin derivatives. Chem. Commun. 2009, 5, 589-591. [CrossRef]

22. Law, H.; Benito, J.M.; Fernández, J.M.G.; Jicsinszky, L.; Crouzy, S.; Defaye, J. Copper(II)-Complex Directed Regioselective Mono-pToluenesulfonylation of Cyclomaltoheptaose at a Primary Hydroxyl Group Position: An NMR and Molecular Dynamics-Aided Design. J. Phys. Chem. B 2011, 115, 7524-7532. [CrossRef]

23. Fuchs, R.; Habermann, N.; Klufers, P. Multinuclear Sandwich-type Complexes of Deprotonated $\beta$-Cyclodextrin and Copper(II) Ions. Angew. Chem. Int. Ed. Engl. 1993, 32, 852-854. [CrossRef]

24. Bagabas, A.A.; Frasconi, M.; Iehl, J.; Hauser, B.; Farha, O.K.; Hupp, J.T.; Hartlieb, K.J.; Botros, Y.Y.; Stoddart, J.F. $\gamma$-Cyclodextrin Cuprate Sandwich-Type Complexes. Inorg. Chem. 2013, 52, 2854-2861. [CrossRef] [PubMed]

25. Díaz, T.E.; Jankowski, C.K.; Hocquelet, C.; del Rio, P.F.; Barrios, H. The unambiguous assignment of NMR spectra of per-Omethylated 6-mono and 6,6-diamino- $\beta$-cyclodextrins. Can. J. Chem. 2008, 86, 726-736. [CrossRef] 